\title{
Un año de Spanish in a day. Resultados y retos futuros
}

\author{
PROVI AYALA CARRASCO \\ Con $\mathrm{C}$ de cine \\ cm@concedecine.com \\ YERAY GONZÁLEZ PLASENCIA \\ Universidad de Salamanca \\ yeraygoncia@usal.es \\ ANTONIO RAMOS ÁLVAREZ \\ Instituto Cervantes de Estambul \\ antonio.ramos@cervantes.es
}

Resumen: en este artículo se ofrecen los lineamientos básicos del proyecto colaborativo Spanish in a day, el primer concurso audiovisual para estudiantes de E/LE de todo el mundo en Internet. En primer lugar, se expone la fase de creación del certamen, atendiendo a las bases, los objetivos, las categorías y la evaluación del mismo. Posteriormente, se muestran las novedades de la segunda edición, poniendo especial énfasis en el concurso paralelo para menores de edad y en el desarrollo de un curso MOOC basado en los vídeos obtenidos en la primera edición. Finalmente, se presentan los resultados del proyecto, profundizando en tres aspectos: los índices de participación, la retroalimentación que se ha obtenido de los participantes y la creación de una película que acoge los vídeos de todos los concursantes.

Palabras clave: concurso audiovisual, nuevas tecnologías, MOOC.

\begin{abstract}
Spanish in a day, the first online video contest for Spanish FL learners worldwide. In the first place, we will describe the key elements when designing the contest (rules, goals, categories and assessment criteria). Subsequently, we will focus on the new features of the $2^{\text {nd }}$ edition, in particular those dealing with the parallel contest for underage students and with the instructional design of a MOOC, based on selected videos of the $1^{\text {st }}$ edition. Lastly, conclusions drawn from data analyses will be addressed in relation to the following aspects: student participation and feedback and the production of a documentary film featuring video clips from all contestants.
\end{abstract}

Keywords: video contest, new technologies, MOOC. 


\section{Introducción}

Spanish in a $\mathrm{day}^{1}$ es un proyecto colaborativo en Internet organizado por la web Con $C$ de Cine, cuyo enfoque está centrado en el alumno como prosumidor de contenidos audiovisuales conforme a los principios básicos del aprendizaje significativo y colaborativo. Asimismo, tiene en cuenta la dimensión afectiva del alumno, ya que le ofrece un marco conceptual altamente motivador, esto es, un concurso a modo de festival de cine internacional en línea cuyo fin último es ganar un curso de español en España. El planteamiento general del concurso consiste en que el estudiante se convierta en el protagonista de un día de su vida y lo explique en español grabándose en un vídeo, imitando las líneas generales de los proyectos in a day, tales como Life in a day o Spain in a day. Aunque los objetivos, la metodología y la aplicabilidad de Spanish in a day difieran de estos proyectos, es innegable la herencia que se percibe de una superproducción como Life in a day en nuestro concurso. Este proyecto audiovisual colaborativo nace con el fin de reflejar cómo es un día cualquiera en la Tierra. Para ello, se invitó a los ciudadanos de todo el mundo a que se grabaran el 24 de julio de 2010, bien llevando a cabo lo que pretendieran hacer ese día o bien contestando a una de las siguientes preguntas: ¿qué hay en tus bolsillos?, ¿qué amas? ¿ ¿a qué le temes? El éxito de Life in a day motivó la aparición de otros proyectos similares de carácter nacional, como Britain in a day, Italy in a day o el ya mencionado Spain in a day.

A diferencia de estos proyectos, en Spanish in a day se ofreció un mayor margen de tiempo para elaborar los vídeos, ya que la prioridad fue que los alumnos pudieran producir minuciosamente tanto sus grabaciones como los guiones de las mismas y que, de este modo, la participación supusiera un aprendizaje para ellos.

Los vídeos enviados por los concursantes presentan muestras reales de estudiantes de E/LE de un valor lingüístico, cultural y pedagógico que ofrecen un sinfín de posibilidades: desde la creación de materiales significativos para el alumno hasta la investigación sobre cualquier aspecto relacionado con la adquisición de lenguas, pasando por el desarrollo de la competencia intercultural en contextos de (no) inmersión lingüística, etc. En definitiva, el proyecto Spanish in a day surge de la necesidad de darle al alumno un mayor protagonismo, siguiendo un enfoque humanista del aprendizaje e integrando las TIC y el uso de las redes sociales para romper las barreras físicas del aula de E/LE.

\section{Spanish in a day I edición}

Spanish in a day se convierte así en el primer concurso audiovisual en Internet para estudiantes de español de todo el mundo. Su primera edición se celebró entre el 1 de abril y el 30 de mayo de 2016, finalizando la votación popular a través de Facebook el 15 de junio de 2016.

Los únicos requisitos para participar en el concurso eran: por una parte, ser estudiante de español como lengua extranjera (E/LE) y, por otra, ser mayor de edad. Siguiendo la premisa de los antecedentes de los proyectos in a day, de los que se ha hablado previamente, el objetivo que se perseguía era que los concursantes grabaran un día en sus vidas, en este caso, como estudiantes de español.

\footnotetext{
${ }^{1}$ Disponible en: www.concedecine.com/spanish-in-a-day/ y www.spanishinaday.com
} 
El concurso se compuso de 3 categorías principales, a las que los participantes accedían una vez realizada una prueba de nivel en línea ${ }^{2}$. Dichas categorías tenían su correlato con los seis niveles del MCER: A de Acción (niveles A1-A2), B de Butaca (niveles B1-B2) y $C$ de Cineclub (niveles C1-C2). Por otra parte, el concurso contaba con dos menciones especiales: $P$ de Producción, dirigida a quienes realizaran un trabajo colectivo, e $I$ de Intercultura, pensada para aquellos que incluyeran un acercamiento entre varias culturas. De este modo, los concursantes podían presentar sus vídeos también a estas menciones para optar a dos becas más. Estos premios consistieron en la financiación de un curso intensivo de castellano en España.

\subsection{Material de apoyo para los estudiantes}

Del mismo modo que en los proyectos in a day se proponía a los participantes una serie de preguntas para motivar la participación, el equipo de Spanish in a day desarrolló un conjunto de materiales cuya finalidad era facilitar la participación en el concurso. Para ello, se tomó como referencia el Plan Curricular del Instituto Cervantes (en adelante, PCIC) (Instituto Cervantes, 2006) y se seleccionaron una serie de estímulos que podían proporcionar ideas a los participantes de cada categoría para contar su historia, como por ejemplo: ¿cuál es tu momento favorito del día? -categoría A de Acción-, ¿tienes alguna habilidad especial? -categoría B de Butaca- o ¿qué cosas haces que te salen "de cine”? - categoría $C$ de cineclub-.

El documento de ayuda -denominado $G$ de guion $^{3}$-, en el que quedaban recogidos tanto estos como otros contenidos útiles para los concursantes, estuvo accesible durante todo el desarrollo del certamen. Además, el jurado valoró positivamente que se recurriera a él y que esto se viera reflejado en las producciones audiovisuales.

\subsection{Evaluación de los vídeos}

Uno de los aspectos del concurso con el que se fue especialmente riguroso fue el de la evaluación de los vídeos, ya que los 15 finalistas del concurso fueron seleccionados gracias a la votación interna que otorgó el jurado experto en evaluación lingüística a cada una de las producciones. Para ello, se diseñaron una serie de herramientas de evaluación (rúbricas e informes compartidos en Google Drive) que permitieron valorar la actuación de los concursantes conforme a un constructo bien identificado que, en la medida de lo posible, fue común a todos los vídeos. En este sentido, el documento $G$ de guion, del que se ha hablado anteriormente, fue de gran utilidad para obtener muestras de contenidos comunicativos de los 6 niveles de referencia del PCIC.

Una de las dificultades con las que nos encontramos fue la necesidad de evaluar a concursantes de una misma categoría principal siguiendo dos rúbricas distintas. Así, en la categoría $A$ de acción valoramos con una rúbrica específica de A1 a aquellos concursantes que habían obtenido ese resultado en la prueba de nivel en línea y con otra

\footnotetext{
${ }^{2}$ La prueba de nivel constaba de 5 bloques de 20 preguntas de selección múltiple. Para garantizar la correcta clasificación de los concursantes en la categoría principal adecuada, estos debían adjuntar el correo con el resultado de la prueba al inscribirse a través de la plataforma oficial del concurso.

${ }^{3}$ Disponible en: www.concedecine.com/wp-content/uploads/2016/04/G-de-guion.pdf.
} 
distinta a los de nivel A2. De este modo, creamos dos rúbricas por cada categoría principal (seis en total), más dos rúbricas ad hoc para evaluar sendas menciones especiales.

Si bien no compartimos expresamente con los concursantes los criterios de evaluación, estos estaban claramente explicados en las bases publicadas en la web oficial del concurso:

(1) los expertos valorarán tu dominio de la lengua, tu interacción con otras personas y la originalidad de la historia en las categorías principales. En las dos menciones especiales tendrán en cuenta la reflexión sobre aspectos culturales y el trabajo en equipo, respectivamente.

Las rúbricas usadas para evaluar tanto las categorías principales como las especiales compartían las mismas 4 bandas de valoración: premio Razzie (1 estrella), ópera prima ( 2 estrellas), peliculón ( 3 estrellas) y obra maestra (4 estrellas). Con estos nombres y con el número de estrellas nos queríamos desvincular de las calificaciones de "apto" y "no apto" empleadas en los DELE, ya que no era nuestro cometido certificar la competencia comunicativa. Además, le estábamos rindiendo un pequeño homenaje al séptimo arte y queríamos poner el foco no tanto en la aptitud del concursante como en la fiabilidad del descriptor de cada banda, independientemente de si recibía 1, 2, 3 o 4 estrellas.

\begin{tabular}{|c|c|c|c|c|c|c|}
\hline NIVEL A2 & VALORACIÓN & 1. Eficacia Lingüística & 2. Eficacia Comunicativa & 3. Eficacia Interactiva & 4. Originalidad & 5. Creatividad \\
\hline$\stackrel{4}{\star \star \star \star}$ & OBRA MAESTRA & $\begin{array}{l}\text { Repertorio lingüistico } \\
\text { suficiente para } \\
\text { comunicarse eficazmente. } \\
\text { Discurso continuo y } \\
\text { cohesionado. }\end{array}$ & $\begin{array}{l}\text { Añade detalles a la } \\
\text { información requerida en el } \\
\text { documento "G de guion" } \\
\text { según su nivel / Transmite los } \\
\text { mensajes en situaciones } \\
\text { cotidianas incluso poniéndolas } \\
\text { en contraste con el pasado. }\end{array}$ & $\begin{array}{l}\text { Se desenvuelve en } \\
\text { situaciones cotidianas } \\
\text { interactuando con soltura } \\
\text { con hispanohablantes, } \\
\text { nativos o no. }\end{array}$ & $\begin{array}{l}\text { Demuestra mucha } \\
\text { originalidad y agrega una } \\
\text { gran variedad de detalles } \\
\text { novedosos en la } \\
\text { producción del video. }\end{array}$ & $\begin{array}{l}\text { Agrega una gran variedad } \\
\text { de detalles para } \\
\text { embellecer su producción } \\
\text { creativa. }\end{array}$ \\
\hline$\stackrel{3}{\star \star \star}$ & ELICULÓN & $\begin{array}{l}\text { Repertorio lingüistico } \\
\text { básico. Errores } \\
\text { sistemáticos, } \\
\text { malentendidos. }\end{array}$ & $\begin{array}{l}\text { Aporta la información } \\
\text { requerida en el doc "G de } \\
\text { guion" según su nivel / } \\
\text { Transmite los mensajes en } \\
\text { situaciones cotidianas. }\end{array}$ & $\begin{array}{l}\text { Ayuda del interlocutor. } \\
\text { Intercambia información e } \\
\text { interactúa con otros } \\
\text { hispanohablantes no } \\
\text { nativos. }\end{array}$ & $\begin{array}{l}\text { Demuestra originalidad y } \\
\text { agrega bastantes detalles } \\
\text { novedosos en la } \\
\text { producción del vídeo. }\end{array}$ & $\begin{array}{l}\text { Agrega bastantes detalles } \\
\text { para embellecer su } \\
\text { producción creativa. }\end{array}$ \\
\hline$\stackrel{2}{\star \star}$ & ÓPERA PRIMA & $\begin{array}{l}\text { Errores frecuentes, } \\
\text { interferencias de otras } \\
\text { lenguas. Discurso muy } \\
\text { limitado. }\end{array}$ & $\begin{array}{l}\text { Datos insuficientes para } \\
\text { comunicarse o hacerse } \\
\text { entender. Se comprenden sólo } \\
\text { fragmentos aislados / No se } \\
\text { basa en el doc "G de guion". }\end{array}$ & $\begin{array}{l}\text { Dependencia del } \\
\text { interlocutor. Dudas, } \\
\text { repeticiones y pausas en la } \\
\text { interacción. }\end{array}$ & $\begin{array}{l}\text { Demuestra cierta } \\
\text { originalidad pero no } \\
\text { agrega detalles novedosos } \\
\text { en la producción del vídeo. }\end{array}$ & $\begin{array}{l}\text { No se observan apenas } \\
\text { detalles para embellecer } \\
\text { su producción creativa. }\end{array}$ \\
\hline $\begin{array}{l}1 \\
\star\end{array}$ & PREMIO "RAZZIE" & $\begin{array}{l}\text { Palabras aisladas, } \\
\text { incomprensibles que } \\
\text { dificultan la comprensión. }\end{array}$ & $\begin{array}{l}\text { No hay apenas comunicación } \\
\text { o la delega en otros actores / } \\
\text { No se basa en el doc "G de } \\
\text { guion" ni en otros contenidos } \\
\text { comunicativos de su nivel. }\end{array}$ & $\begin{array}{l}\text { Dependencia total del } \\
\text { interlocutor: reformulaciones } \\
\text { y repeticiones. Apenas } \\
\text { interactúa con otros. }\end{array}$ & $\begin{array}{l}\text { Demuestra muy poca } \\
\text { originalidad y no agrega } \\
\text { detalles novedosos en la } \\
\text { producción del vídeo. }\end{array}$ & $\begin{array}{l}\text { No se observa ningún } \\
\text { detalle para embellecer su } \\
\text { producción creativa. }\end{array}$ \\
\hline
\end{tabular}

Imagen 1. Rúbrica para la valoración de los vídeos presentados a la categoría A de acción

Para diseñar las rúbricas de las categorías principales se tomaron como punto de partida las escalas holísticas de los DELE. Así pues, los tres primeros criterios fueron la eficacia lingüistica, la eficacia comunicativa y la eficacia interactiva. En cuanto al segundo criterio, además de los descriptores del DELE para las cuatro bandas, añadimos un descriptor alternativo mediante el cual evaluábamos si el concursante se había basado en el documento $G$ de guion y en qué medida se ajustaba o no a los contenidos de dicho documento. Con respecto a la eficacia interactiva, decidimos valorar de diferente manera si la interacción se llevaba a cabo con hablantes nativos o no. De esta forma, la interacción adecuada con hablantes nativos recibía una valoración de obra maestra, mientras que la interacción adecuada con hablantes no nativos recibía una valoración de peliculón.

Como puede observarse en la imagen 1, la creatividad y la originalidad eran los otros dos criterios que completaban las rúbricas, siendo las cuatro bandas comunes a los seis niveles. Para su creación, se tomó como referencia el Test of Creative Thinking (Torrance, 1966), en el que se propone medir, por un lado, la originalidad -en función del número de respuestas novedosas no convencionales que aporta el concursante- $y$, por otro, la 
creatividad -en función de la cantidad de detalles que embellecen y mejoran la producción-.

A fin de validar las rúbricas antes de utilizarlas en la fase de evaluación, se llevó a cabo un pilotaje previo con vídeos similares a los del concurso, con el propósito de ilustrar con muestras reales los descriptores de las cuatro bandas y consensuar así buenas prácticas y homogeneizar los criterios de evaluación.

\section{Spanish in a day II edición: retos y novedades}

La segunda edición del concurso se desarrolló entre el 17 de marzo y el 16 de junio de 2017. En ella, se mantuvieron las categorías principales, pero se eliminaron las menciones $P$ de Producción -ya que uno de los requisitos es que se participe en grupo -e $I$ de Intercultura -puesto que todos los vídeos debían incluir una reflexión crítica intercultural-.

\subsection{Spanish in a day Junior y mención especial Welcome}

En la primera edición de Spanish in a day se recibieron contribuciones de estudiantes de E/LE menores de edad. Sus profesores habían incorporado el concurso a su proyecto docente $\mathrm{y}$, aunque sabían que no podían participar por no cumplir con uno de los requisitos de las bases, quisieron hacernos llegar las producciones de sus alumnos. Este hecho, junto al interés que despertó la iniciativa en foros de E/LE entre docentes de colegios e institutos, motivó que se creara Spanish in a day Junior, un concurso paralelo para estudiantes de E/LE de 14 a 17 años.

Por otra parte, el equipo Spanish in a day es sensible a la crisis que está viviendo nuestra sociedad en cuanto a las personas refugiadas y solicitantes de asilo. Ante esta realidad, se desarrolló una mención del concurso para todos aquellos estudiantes de E/LE que tuvieran este estatus reconocido en España, dando lugar a Spanish in a day Welcome.

Tanto el concurso paralelo Junior como la mención Welcome se llevaron a cabo bajo los mismos criterios de participación y evaluación que el resto del certamen. En el caso del concurso Junior, existen las tres categorías principales y la mención I de Intercultura, mientras que la sección Welcome se integra en el concurso para adultos. En resumen, el objetivo fue dar la posibilidad de participar a dos colectivos para los que consideramos que Spanish in a day puede ser una buena herramienta pedagógica.

\subsection{Spanish in a day NanoMOOC}

En la primera edición del concurso se detectó que, en ocasiones, los vídeos enviados no respetaban las pautas recomendadas, a pesar del esfuerzo realizado en transmitirlas a través de la guía didáctica y, especialmente, del documento $G$ de guion. Esta carencia se transformó en reto y el equipo de Spanish in a day decidió apostar por una formación más integral en su segunda edición mediante la creación del curso NanoMOOC.

El Instituto Nacional de Tecnologías Educativas y de Formación del Profesorado (INTEF, s. f.) define los NOOC (NANO Curso Abierto, Masivo y En Línea, NOOC en sus siglas en inglés) como una herramienta que 
le da a los participantes la oportunidad de explorar, aprender y ser evaluados sobre un elemento clave de una competencia, una destreza, o un área de conocimiento en un periodo de tiempo que puede ir desde un mínimo de 1 hora hasta un máximo de 20 horas de esfuerzo estimado de dedicación.

(INTEF, s. f.).

Spanish in a day NanoMOOC fue un curso gratuito, cuyos materiales estuvieron disponibles ${ }^{4}$ hasta la finalización de la segunda edición (abierto); se diseñó para un número ilimitado de participantes (masivo) y se administró en su totalidad a través de la Red (en línea). Además, se consideró un esfuerzo estimado para su realización de 10 horas, empleadas en adquirir las destrezas y las estrategias necesarias para crear una producción audiovisual acorde a los contenidos comunicativos del nivel de lengua de los participantes y a los requisitos propios del concurso.

Finalmente, el curso se concibió en función de otro de los presupuestos teóricos fundamentales de los NOOC, esto es, una modularidad en los contenidos que garantice el aprendizaje flexible y motivador del alumno (Godwin-Jones, 2014). Así pues, Spanish in a day NanoMOOC constó de tres itinerarios conforme a las tres categorías principales del concurso ( $A$ de Acción, B de Butaca y $C$ de Cineclub), compuesto cada uno por siete módulos, de los cuales cuatro fueron comunes y tres de ellos exclusivos para cada itinerario.

Los tres módulos propios de cada itinerario fueron aquellos vinculados al nivel de lengua de los estudiantes, e incluían: contenidos comunicativos y lingüísticos (segundo módulo), estrategias de comunicación verbal y no verbal para un discurso eficaz (tercero) y elaboración de un guion cinematográfico (séptimo módulo y tarea final). Los módulos comunes fueron: objetivos y presentación del curso (primer módulo), estrategias para la producción creativa y original (cuarto), pautas para una producción colectiva (quinto) y destrezas interculturales (sexto).

\section{Resultados de Spanish in a day}

\subsection{Spanish in a day, I edición}

En la I edición de Spanish in a day se recibieron 43 inscripciones: 31 en las tres categorías principales y 12 en las menciones especiales I de Intercultura y P de Producción. En total, se registraron 55 participantes de 18 países $^{5}$ y 4 continentes distintos, entre actores, cámaras, guionistas, editores, etc. Resulta muy llamativo que de todas las inscripciones recibidas, solo 6 de ellas se correpondieran con vídeos realizados en contexto de inmersión lingüística (España).

De todos los vídeos recibidos, 15 de ellos resultaron finalistas y 5 de ellos se alzaron con el premio en su categoría correspondiente. Ningún vídeo resultó premiado en más de una categoría, lo que da fe de la gran calidad de las inscripciones recibidas y de lo reñidas que estuvieron las votaciones en Facebook hasta el último momento.

\footnotetext{
${ }^{4}$ Alojados en la página http://spanishinaday.com/nanomooc.

5 Los países de origen de los concursantes fueron: Afganistán, Alemania, Argelia, Bielorrusia, Brasil, China, Estados Unidos, Estonia, Grecia, Hong Kong, Italia, Japón, Marruecos, Rusia, Taiwán, Turquía, Noruega y Vietnam.
} 


\subsection{Spanish in a day, la peli}

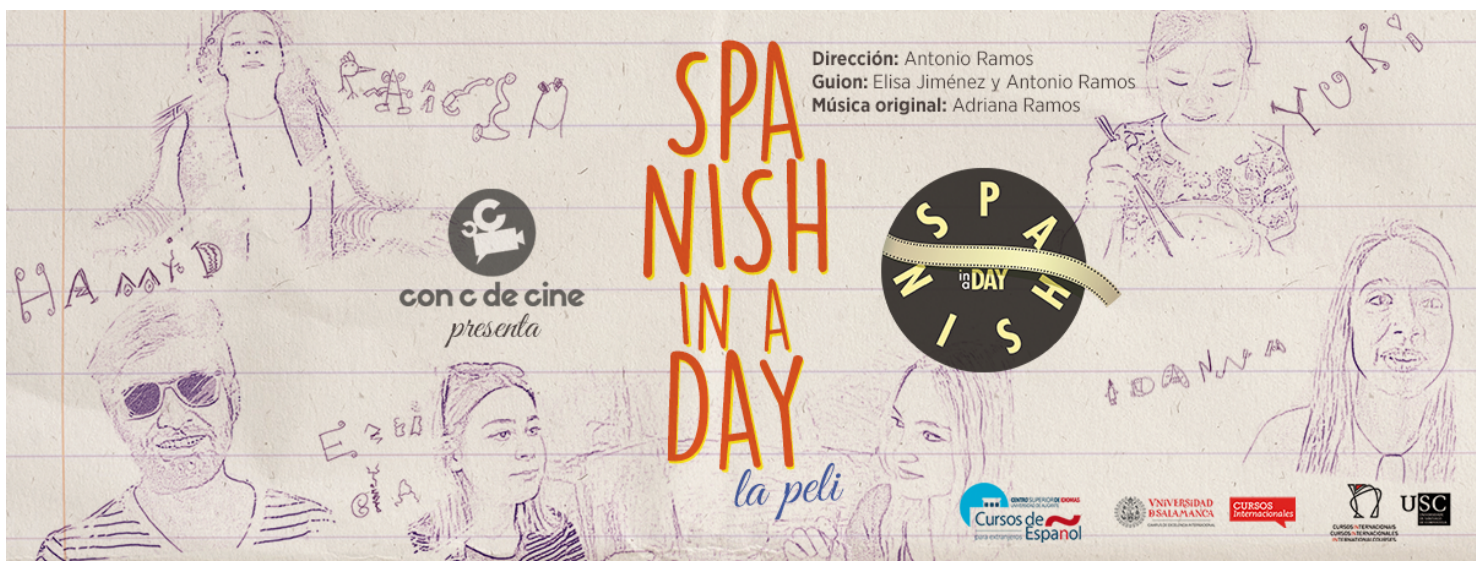

Imagen 2. Cartel de Spanish in a day, la peli (2017)

El documental Spanish in a day, la peli ${ }^{6}$ surgió con el propósito de dar voz a todos los concursantes de la I edición y de crear un producto audiovisual que resumiera los mejores momentos de las contribuciones. Para ello, se tomaron como modelo los proyectos Life in a day y Spain in a day, y se seleccionaron las escenas que por su calidad pedagógica y audiovisual merecían formar parte de la película. Finalmente, debido a la cantidad y calidad del material recibido, el documental se dividió en 3 cortometrajes de 6 minutos cada uno. Estas tres piezas están agrupadas por áreas temáticas y siguen una narrativa intercultural: la rutina diaria, los estudios y el trabajo, la vida en la ciudad, el ocio y el tiempo libre, la comida y la gastronomía, los gustos e intereses, el clima y la influencia del mar en la vida de las personas, etc.

Como se observa en la imagen 3, los tres cortometrajes se dividen en 5 o 6 piezas de un minuto cada una (16 en total), las cuales están publicadas en la web de la II edición del concurso junto a sus respectivas guías didácticas. Gracias a su corta duración, estos fragmentos están publicados asimismo en Facebook, ya que se adaptan muy bien al tipo de contenidos audiovisuales y a los hábitos de consumo de los usuarios de las redes sociales. Por su parte, los tres cortometrajes y la película entera se han proyectado en varios congresos y jornadas de formación de profesores nacionales e internacionales desde su estreno ${ }^{7}$.

\begin{tabular}{|c|c|c|}
\hline ¡Buenos días... Buenas noches! & De paseo y de tapeo & Vamos a pasarlo bien \\
\hline ¡Hola! ¿Qué tal? & Ciudades del mundo & ¡Cómo me gusta! \\
\hline ¡Buenos días! & ¡Vamos de paseo! & Llueve a mares \\
\hline
\end{tabular}

\footnotetext{
${ }^{6}$ Tanto los teaser como las guías didácticas que incluyen las transcripciones de los vídeos están disponibles en http://spanishinaday.com/la-peli/spanish-in-a-day-la-peli

${ }^{7}$ La película se estrenó el 1 de marzo de 2017. Para más información sobre los pases del documental, pueden consultar la "Agenda de formación" de Con C de Cine, disponible en www.concedecine.com/p-deprofesor/agenda.
} 


\begin{tabular}{|c|c|c|}
\hline ¿Estudias o trabajas? & ¡A comer! & Un día de ocio \\
\hline Estudio español & ¡Qué rico! & ¿Quedamos esta noche? \\
\hline ¡Buenas tardes! & ¡Que aproveche! & ¡Hasta la próxima! \\
\hline ¡Buenas noches! & & \\
\hline
\end{tabular}

\subsection{Spanish in a day, II edición}

En la segunda edición del concurso participaron 170 estudiantes de 30 nacionalidades diferentes, los cuales representaron a cuatro continentes: África, América, Asia y Europa. Por lo que respecta a la segunda fase del concurso, esto es, la difusión de los vídeos finalistas en las redes sociales, se registraron más de 600000 personas alcanzadas, con un total de 45000 interacciones en 22 idiomas.

Han de destacarse por otra parte, por su valor pedagógico, los resultados del curso de preparación Spanish in a day NanoMOOC, el cual, como ya se expuso anteriormente, tuvo lugar entre el 31 de marzo y el 23 de abril de $2017^{8}$.

Durante las 5 semanas comprendidas entre el lanzamiento del concurso (17 de marzo) y la finalización del curso ( 23 de abril), el registro de la página web donde se alojaba el curso recibió más de 700 inscripciones. De estos usuarios, 141 comenzaron el curso y 61 lo terminaron según los criterios de evaluación establecidos, lo que supone un $43 \%$ del total. Si se tiene en consideración que las tasas de finalización en los MOOC suelen ser inferiores al 13\% (Onah, Sinclair y Boyatt, 2014), los resultados fueron muy satisfactorios.

Conviene señalar que, aunque el curso estaba dirigido principalmente a estudiantes de ELE, hubo bastantes docentes de español que lo realizaron con el objetivo de preparar a sus propios alumnos según las bases y los criterios de evaluación de la categoría correspondiente a su nivel de lengua. El siguiente ejemplo, extraído del cuestionario de evaluación que debían completar los participantes al final del curso, ilustra lo expuesto:

(2) Recomendaré a mis alumnos estrategias relacionadas con la elección de un entorno que resulte estimulante e informativo, la preparación de un guion y dibujos de la situación comunicativa, la introducción de elementos que fomenten la creatividad, la interculturalidad, el interés y la tolerancia y el uso de un lenguaje no verbal atractivo.

Rafael, profesor de E/LE, tarea final, NanoMOOC Acción

En cuanto a los estudiantes que realizaron el curso con el fin de prepararse para participar en el certamen, cabe destacar la gran cantidad de jóvenes de origen italiano que terminaron el NanoMOOC Butaca. Estos datos apuntan a que probablemente haya un mayor número de inscripciones en el concurso Junior de vídeos correspondientes a los niveles B1-B2:

\footnotetext{
${ }^{8}$ Una vez terminado el curso, los materiales siguen estando disponibles hasta el 28 de mayo (plazo para el envío de los vídeos al concurso). Igualmente, se puede acceder a los foros de los 3 cursos a través de la siguiente dirección: http://spanishinaday.com/forums.
} 
(2) ¡Hola! Me llamo Dalila, soy italiana y vivo en un pequeño pueblo cerca de Lecce. Tengo 16 años. Me gusta mucho aprender idiomas estranjeras (sic), viajar, practicar el baloncesto con amigos. Estudio español desde hace 3 años y es mi lengua favorita. Me gustaría mejorar mi manera de hablar en español con este curso.

Dalila, estudiante de ELE, foro de presentaciones, NanoMOOC Butaca

\section{Conclusiones}

Spanish in a day ha supuesto una forma novedosa y muy motivadora de aprender español, puesto que ha fomentado el trabajo colaborativo entre alumnos y profesores cuestionando las barreras físicas y metodológicas del aula de E/LE. El siguiente testimonio ${ }^{9}$ da fe de ello:

(3) El concurso Spanish in a day ha sido genial. Es una manera diferente de aprender español. Hacer el vídeo fue muy divertido gracias a la ayuda de mi profesor y mis amigos. Después, ver los vídeos de otros estudiantes del mundo en Facebook fue también muy interesante.

Hamid, ganador de la categoría A de Acción

Por otra parte, esta iniciativa ha contribuido al desarrollo personal de los estudiantes, ya que la experiencia de aprendizaje no se limitaba solamente a la fase de producción de los vídeos y a la posterior interacción en español en las redes sociales. En última instancia, los concursantes ganadores disfrutaron de una beca de estudios en una ciudad española, por lo que su esfuerzo se vio merecidamente recompensado. Más allá del premio en sí, la estancia enriqueció notablemente la dimensión personal del estudiante y fomentó su conciencia intercultural:

(4) Fue una experiencia increíble en todos los sentidos. Durante mi estancia en Madrid tuve la oportunidad de conocer en primera persona la cultura española, interactuar con hablantes nativos y mejorar no solo mi español sino también mi modo de pensar. (Ioanna, ganadora de la categoría $C$ de Cineclub).

Además, Spanish in a day ha servido para establecer sinergias de colaboración muy fructíferas entre entidades públicas y privadas del ámbito de E/LE, lo cual redunda en la calidad de la enseñanza recibida por parte de los estudiantes:

(5) Además, los profesores de la Universidad de Santiago son muy buenos y me ayudaron mucho. Las clases eran muy activas y dinámicas, siempre trabajábamos en grupos pequeños y aprendimos mucho de la cultura de Galicia y España.

Hamid, Afganistán, ganador de $A$ de Acción

(6) En IMSOL los profesores son simpáticos y tienen un excelente método de enseñanza. Me gustan mucho las clases de cultura y las actividades extras son fundamentales para la absorción de los contenidos.

Raissa, Brasil, ganadora de $B$ de Butaca

(7) He tenido una experiencia fantástica en la Universidad de Salamanca. Los profesores han sido muy amables conmigo.

Yuki, Hong Kong, ganador de I de Intercultura

\footnotetext{
${ }^{9}$ Los testimonios íntegros de los ganadores del concurso están disponibles en la siguiente página web: http://www.concedecine.com/e-de-estudiante/spanish-in-a-day/testimonios.
} 
Finalmente, queremos realzar la repercusión pedagógica del curso NanoMOOC. Consideramos que esta experiencia de aprendizaje ha proporcionado a los estudiantes tanto las herramientas y estrategias necesarias para producir contenido audiovisual en español conforme a las bases del concurso como los contenidos lingüísticos, comunicativos y culturales que complementan, sin lugar a duda, la formación que reciben en sus instituciones. Todo ello a través de muestras reales de hablantes que tienen el español como una de sus lenguas extranjeras. En conclusión, Spanish in a day supone una reinterpretación del estímulo que se le ofrece al estudiante de E/LE, ya que se ajusta a las necesidades del alumnado actual, inmerso en un mundo cambiante, multicultural y polifónico.

\section{Bibliografía}

GODWIN-JONES, R. (2014). «Global reach and local practice: The promise of MOOCS», Language Learning \& Technology, 18(3), 5-15.

INTEF (s. f.). ¿Qué es un NOOC?, Recuperado el 6/5/2017, de: http://educalab.es/intef/formacion/formacion-en-red/nooc.

Instituto Cervantes (2006). Plan curricular del Instituto Cervantes. Niveles de referencia para el español. Madrid: Instituto Cervantes-Biblioteca Nueva.

ONAH, D. F. O., Sinclair, J. y BOYATT, R. (2014). «Dropout rates of massive open online courses: behavioural patterns». En 6th International Conference on Education and New Learning Technologies, Barcelona, Spain, 7-9 Jul 2014, EDULEARN14 Proceedings (pp. 5825-5834). Recuperado el 4/5/2017 de http://wrap.warwick.ac.uk/65543/

TORRAnCE, E. P. (1966). Torrance Tests of Creative Thinking. Lexington, MA: Personnel Press. 